\title{
Cardiovascular Profile of Patients with Type 2 Diabetes in France Based on REWIND CVOT Inclusion Criteria: A Real-World Retrospective Study
}

\author{
Anne Dib · Florence Chartier · Katharina Ihle · Isabelle Jamonneau • \\ Lisa Ludwig · Patrice Darmon
}

Received: November 17, 2021 / Accepted: December 24, 2021 / Published online: January 15, 2022

(C) The Author(s) 2022

\begin{abstract}
Introduction: The REWIND study demonstrated a cardiovascular (CV) benefit of dulaglutide treatment in patients with type 2 diabetes (T2D) with or without established cardiovascular disease (CVD). The current study aims to describe similarities and differences between characteristics of patients with T2D in France and the REWIND population.

Methods: A retrospective, observational study was conducted in France using primary care IQVIA electronic medical records. Patients aged $\geq 18$ years with at least one clinical visit and/or glucose-lowering agent prescription in 2019 were identified. The percentages of patients aged $\geq 50$ years with established CVD, aged $\geq 55$ years with subclinical CVD or aged $\geq$
\end{abstract}

Supplementary Information The online version contains supplementary material available at https:// doi.org/10.1007/s13300-021-01200-0.

A. Dib $(\varangle)$ · F. Chartier · I. Jamonneau · L. Ludwig Eli Lilly France SAS, Neuilly Sur Seine, France e-mail: dib_anne@lilly.com

K. Ihle

Eli Lilly Deutschland GmbH, Bad Homburg,

Germany

P. Darmon

Aix Marseille University, INSERM, INRA, C2VN,

Marseille, France
60 years with multiple CV risk factors based on REWIND definitions were calculated.

Results: A total of 63,927 patients with T2D were included. Mean age was 67 years, 93\% were aged $\geq 50$ years and $58 \%$ were male. The median time since T2D diagnosis was 5.6 years, mean glycated hemoglobin was $7.1 \%$ and mean body mass index was $30.4 \mathrm{~kg} / \mathrm{m}^{2}$. Of the patients included in the current study, $59.4 \%$ fulfilled REWIND CV criteria; $12.4 \%$ of patients were $\geq 50$ years old with established CVD; $9.7 \%$ of patients were aged $\geq 55$ years with subclinical vascular disease and $44.7 \%$ were aged $\geq 60$ years with $\geq 2 \mathrm{CV}$ risk factors.

Conclusion: Almost $60 \%$ of this primary care French cohort with T2D fulfilled key REWIND $\mathrm{CV}$ criteria, with a lower percentage of patients having established CVD than REWIND participants.

Keywords: Cardiovascular disease; France; Primary care; Type 2 diabetes 


\section{Key Summary Points}

Why carry out this study?

The REWIND cardiovascular outcomes trial demonstrated the benefit of the glucagon-like peptide-1 receptor agonist dulaglutide in reducing major adverse cardiovascular $(\mathrm{CV})$ events in patients with type 2 diabetes (T2D) aged $\geq 50$ years with established or at risk of CV disease.

This study uses real-world clinical data to describe the characteristics of patients with T2D in routine primary care in France based upon key inclusion criteria of the REWIND study to assess the proportion of T2D patients in France eligible for REWIND according to their CV profile.

\section{What was learned from the study?}

Electronic medical records from the IQVIA database were used to access data from patients with T2D in primary care in France between 2009 and 2019.

Patient characteristics, such as age, glycated hemoglobin and body mass index were comparable between the study population and REWIND participants.

Approximately $60 \%$ of the study population fulfilled key REWIND CV inclusion criteria; $12.4 \%$ of patients were aged $\geq 50$ years with established $\mathrm{CV}$ disease, $9.7 \%$ were aged $\geq 55$ years with subclinical vascular disease and $44.7 \%$ of patients were aged $\geq 60$ years with $\geq 2 \mathrm{CV}$ risk factors.

\section{INTRODUCTION}

Cardiovascular disease (CVD) persists as a major comorbidity for patients with type 2 diabetes (T2D) [1]. Several glucagon-like peptide-1 receptor agonists (GLP-1 RA) have demonstrated a beneficial cardiovascular (CV) effect in patients with T2D and established CVD or cardiovascular $(\mathrm{CV})$ risk factors [2]. However, GLP-1 RA cardiovascular outcomes trials (CVOTs) mainly included patients with established CVD [3-7], with the exception of the REWIND study which also included a substantial proportion of patients with no history of established CVD $[2,8]$. The REWIND CVOT demonstrated the benefit of the GLP-1 RA dulaglutide in reducing major adverse $\mathrm{CV}$ events in patients with T2D aged $\geq 50$ years with or without established CVD [9]. Key inclusion criteria were (1) a baseline glycated hemoglobin $(\mathrm{HbA} 1 \mathrm{c}) \leq 9.5 \%$; $(2)$ receiving up to two oral glucose-lowering agents (GLA) with or without basal insulin; (3) and aged $\geq 50$ years with established CVD, aged $\geq 55$ years with subclinical CVD or aged $\geq 60$ years with $\geq 2 \mathrm{CV}$ risk factors [10]. During the median follow-up of 5.4 years, dulaglutide treatment demonstrated a significantly lower risk of the primary composite endpoint of non-fatal myocardial infarction, non-fatal stroke or CV death compared to placebo (hazard ratio 0.88; 95\% confidence interval 0.79-0.99) $[9,11]$. Despite the considerable impact of CVOTs, limited data are available on the generalizability of results due to restrictive inclusion and exclusion criteria in all CVOTs conducted with antidiabetic drugs. A USA-based study examined the extent to which GLP-1 RA CVOTs were generalizable to the overall T2D population [8]. The results suggested that the characteristics of the patients with T2D included in the REWIND study were the most similar to those in the US adult T2D population than those of other GLP-1 RA CVOTs. In France, the ENTRED public health survey is periodically conducted on a national representative sample of people living with diabetes in order to accurately characterize their health status, the quality of care they received and the evolution trend of these parameters. The most recent assessment from 2007 indicated that a majority of patients with T2D had a very high risk of CVD, but the proportion of patients who would have fulfilled the REWIND CV inclusion criteria is unknown $[9,11,12]$.

The aim of the current study was to describe similarities and differences in patient 
characteristics between a cohort of patients with T2D followed in routine primary care in France and the T2D population included in the REWIND study, and to define the prevalence of established CVD in the French cohort using similar definitions to those used in the REWIND study.

\section{METHODS}

\section{Database}

A retrospective, observational study was conducted in France using IQVIA's Electronic Medical Records (EMR) database contained in the Longitudinal Patient Data (LPD) IQVIA tool. The LPD tool contains anonymized prescription, patient and medical information from Patient Management software used by primary care physicians (general practitioners [GPs]) and specialists. The database is considered representative of day-to-day clinical practices as it details patient information and physicians' care without any interventional purpose. The panel of physicians is representative of the primary care physician according to three criteria known to influence prescribing: age, gender and geographical distribution. For this study, only the GP panel was used $(N=1200)$ as the population of interest were patients with T2D, and these patients are mainly followed by GPs in routine care. Additionally, no unique identifier existed to link patient data from GPs and specialists and, therefore, not including patients treated by specialists prevented a duplication of patient data. Data collected included sociodemographic information, medical information (including T2D diagnosis details, comorbidities, history of CVD) and T2D treatment details. Diagnoses of T2D, CVD comorbidities, and other comorbidities were coded based on the International Classification of Diseases (ICD)-10 system. This study is based on de-identified data collected from a healthcare database. According to the French law no submission to CESREES (Ethics and Scientific Committee for Research, Studies and Evaluations in the Field of Health) was required. This study complied with the reference method MR004 of CNIL (French data privacy agency). As required, the study was recorded for information on the French Health Data Hub website.

\section{Study Design}

The study inclusion period extended from 1 January 2019 to 31 December 2019. Patients aged $\geq 18$ years with previously or newly diagnosed T2D were identified. Eligible patients had at least one visit with T2D diagnosis and/or at least one prescription of a glucose-lowering agent during the inclusion period. If not diagnosed during the inclusion period, patients were required to have a record of T2D diagnosis within the preceding 10 years (from 1 January 2009). A 24-month pre-index period from 1 January 2017 to 1 January 2019 was included to assess patient and clinical characteristics. A 3-month follow-up window from 31 December 2019 was included to assess laboratory results.

\section{Patient Criteria}

Patients included in the study were aged $\geq$ 18 years at the index date and fulfilled diagnosis or prescription, and medical history requirements detailed in Study Design. Patients were excluded if they had a diagnosis of type 1 diabetes among back-data prior to the index date or had gestational diabetes or were pregnant in the 9 months before or at the index date.

Patients aged $\geq 50$ years at the index date were identified as a subgroup of interest due to the REWIND CV inclusion criteria [10]. Among these patients, two distinct groups were categorized following the REWIND enrollment criteria: (1) those aged $\geq 50$ years with a history of established CVD and (2) those with high CV risk but without a history of established CVD-either aged $\geq 55$ years with subclinical vascular disease or aged $\geq 60$ years with multiple CV risk factors. A history of established CVD was defined as prior myocardial infarction, ischemic stroke, presence of coronary revascularization, occlusion of stenosis of precerebral arteries or carotid artery and/or hospitalization for unstable angina. See Electronic Suplementary Material (ESM) Table S1 for inclusion criteria of the 
REWIND study and of the current study with proxies used in the current analysis.

Comorbidities of interest were identified within the database with all back-data period available (i.e., up to 10 years), except for obesity which was identified within the 24 months prior to the index date. Comorbidities were identified from physician reports in the database. However, to address potential underreporting of the comorbidities hypertension and dyslipidemia, additional analyses were done to identify patients with hypertension and/or prescription of blood pressure-lowering medication, and those with hyperlipidemia and/or prescription of lipid-lowering medications.

\section{Statistical Analysis}

Descriptive analysis was used. For the baseline characteristics analysis, the REWIND population was described using published data only $[9,10]$. Presented characteristics included age, sex, body mass index (BMI), duration of diabetes, HbA1c and other key laboratory and diagnostic variables.

Proxies for key REWIND CV criteria were applied to the general T2D study population, as described in Study Design. Continuous variables were described by number of values, mean and standard deviation (SD), or median with 1st and 3rd quartile. Categorical variables were described by the number of values, the frequency and the percentage of each modality. All results were presented with one decimal. All analyses were performed using SAS $\AA$ enterprise guide 6.1 (SAS 9.4) software (SAS Institute, Cary, NC, USA).

\section{RESULTS}

Of the T2D primary care population in the EMR database, 87,670 patients with a diagnosis of T2D and/or at least one prescription of a glucose-lowering agent were identified during the inclusion period. Of these, 63,927 (72.9\%) satisfied the inclusion and exclusion criteria (Fig. 1) and were classified as the general T2D study population. Most baseline characteristics of the study cohort were comparable with those

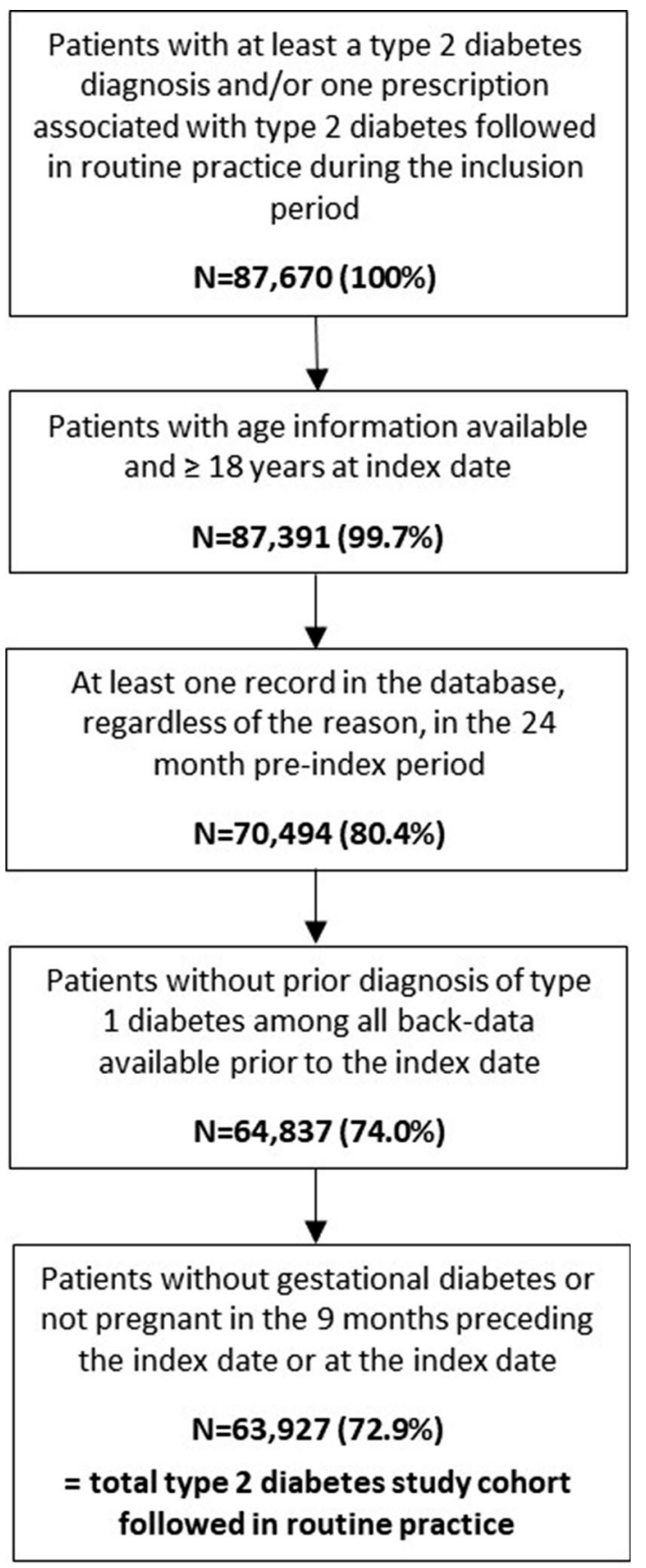

Fig. 1 Flow chart of patient inclusion in analysis cohort

of the REWIND participants (Table 1). However, some differences were observed between the populations, such as diabetes duration and comorbidities.

The mean $( \pm \mathrm{SD})$ age of the study cohort was 67 ( \pm 11.7$)$ years, and 59,313 (92.8\%) were aged $\geq 50$ years, $58.2 \%$ were male, mean HbA1c was 
Table 1 Baseline characteristics of total type 2 diabetes study cohort, those aged $\geq 50$ years fulfilling key REWIND cardiovascular criteria and REWIND participants

\begin{tabular}{|c|c|c|c|c|c|}
\hline $\begin{array}{l}\text { Baseline } \\
\text { characteristics }\end{array}$ & $\begin{array}{l}\text { Total T2D } \\
\text { study cohort } \\
(N=63,927)\end{array}$ & $\begin{array}{l}\text { Total T2D population } \\
\text { fulfilling key REWIND CV } \\
\text { criteria }(N=37,965)\end{array}$ & $\begin{array}{l}\text { Population without } \\
\text { established CVD } \\
(N=30,043)\end{array}$ & $\begin{array}{l}\text { Population with } \\
\text { established CVD } \\
(N=7922)\end{array}$ & $\begin{array}{l}\text { REWIND } \\
\text { patients } \\
(N=9901)\end{array}$ \\
\hline Age, years & $67.0(11.7)$ & $71.6(8.2)$ & $71.7(7.8)$ & $71.4(9.5)$ & $66.2(6.5)$ \\
\hline Males, $n(\%)$ & $37,177(58.2)$ & $22,470(59.2)$ & $16,635(55.4)$ & $5835(73.7)$ & $5312(53.7)$ \\
\hline BMI, $\mathrm{kg} / \mathrm{m}^{2}$ & $30.4(5.8)^{\mathrm{b}}$ & $30.5(5.5)^{\mathrm{b}}$ & $30.8(5.6)^{\mathrm{b}}$ & $29.5(5.2)^{\mathrm{b}}$ & $32.3(5.7)$ \\
\hline $\begin{array}{l}\text { T2D duration, } \\
\text { years }\end{array}$ & $5.6(3.5)$ & $6.5[3.0,9.8]$ & $6.5[3.0,9.8]$ & $6.8[3.2,9.9]$ & $\begin{array}{c}9.5[5.5 \\
14.5]\end{array}$ \\
\hline HbAlc, \% & $7.1(1.3)^{\mathrm{b}}$ & $7.1(1.2)^{\mathrm{b}}$ & $7.1(1.2)^{\mathrm{b}}$ & $7.2(1.2)^{\mathrm{b}}$ & $7.3(1.1)$ \\
\hline \multicolumn{6}{|l|}{ Hypertension, $n$ (\%) } \\
\hline $\begin{array}{l}\text { Diagnosed } \\
\text { comorbidity }\end{array}$ & $40,815(63.8)$ & $28,451(74.9)$ & $22,850(76.1)$ & $5601(70.7)$ & $9223(93.2)$ \\
\hline $\begin{array}{l}\text { Antihypertensive } \\
\text { agent }(s)\end{array}$ & $46,929(73.4 \%)$ & $33,446(88.1 \%)$ & $26,226(87.3 \%)$ & $7220(91.1 \%)$ & NA \\
\hline $\begin{array}{l}\text { Albuminuria } \\
\quad \text { diagnosis, } n(\%)^{a}\end{array}$ & $1001(1.6)$ & $915(2.4)$ & $700(2.3)$ & $215(2.7)$ & $3467(35.0)$ \\
\hline $\begin{array}{l}\text { eGFR, } \mathrm{mL} / \mathrm{min} / \\
1.73 \mathrm{~m}^{2}\end{array}$ & $80.7(24.3)^{\mathrm{b}}$ & $76.4(23.7)^{\mathrm{b}}$ & $76.6(23.2)^{\mathrm{b}}$ & $75.5(25.4)^{\mathrm{b}}$ & $76.9(22.7)$ \\
\hline $\begin{array}{l}\mathrm{eGFR}<60 \mathrm{~mL} / \\
\min / 1.73 \mathrm{~m}^{2}, n \\
(\%)\end{array}$ & $1876(18.0)^{\mathrm{b}, \mathrm{c}}$ & $1788(23.1)^{\mathrm{b}, \mathrm{c}}$ & $1353(22.3)^{\mathrm{b}, \mathrm{c}}$ & $435(26.3)^{\mathrm{b}, \mathrm{c}}$ & $2199(22.2)$ \\
\hline $\begin{array}{l}\text { LDL cholesterol, } \\
\mathrm{mmol} / \mathrm{L}\end{array}$ & $2.6(1.0)^{\mathrm{b}}$ & $2.5(1.0)^{\mathrm{b}}$ & $2.6(1.0)^{\mathrm{b}}$ & $2.2(1.0)^{\mathrm{b}}$ & $2.6(1.0)$ \\
\hline $\begin{array}{l}\text { HDL cholesterol, } \\
\mathrm{mmol} / \mathrm{L}\end{array}$ & $1.3(0.4)^{\mathrm{b}}$ & $1.3(0.4)^{\mathrm{b}}$ & $1.3(0.4)^{\mathrm{b}}$ & $1.2(0.4)^{\mathrm{b}}$ & $1.2(0.3)$ \\
\hline $\begin{array}{l}\text { Triglycerides, } \\
\mathrm{mmol} / \mathrm{L}\end{array}$ & $1.5[1.1,2.2]^{\mathrm{b}}$ & $1.6[1.1,2.2]^{\mathrm{b}}$ & $1.6[1.2,2.2]^{\mathrm{b}}$ & $1.5[1.1,2.1]^{\mathrm{b}}$ & $1.6[1.2,2.2]$ \\
\hline
\end{tabular}

All data in table are presented as the mean with the standard deviation (SD) in parenthesis or as the median with the interquartile range [Q1, Q3] given in square brackets unless otherwise indicated

$B M I$ Body mass index, eGFR estimated glomerular filtration rate, $H b A l c$ glycated hemoglobin Alc, $H D L$ high-density lipoprotein, $L D L$ low-density lipoprotein, $N$ number of patients, $N A$ not available, $Q 1$ 1st quartile, $Q 33$ rd quartile, $S D$ standard deviation, $T 2 D$ type 2 diabetes

${ }^{2}$ Data were collected as categorical variables yes/no which did not allow missing data to be identified

${ }^{\mathrm{b}}$ Total T2D study cohort, total population fulfilling key REWIND criteria, those without established CVD and those with established CVD with available data, respectively, were: BMI: 27,216 (42.6\%), 18,283 (48.2\%), 14,543 (48.4\%), 3,740 (47.2\%); eGFR: 10,402 (16.3\%), 7,734 (20.4\%), 6,080 (20.2\%), 1,654 (20.9\%); HbAlc: 27,366 (42.8\%), 19,016 (50.1\%), 15,200 (50.6\%), 3,816 (48.2\%); HDL cholesterol: 20,800 (32.5\%), 15,222 (40.1\%), 12,233 (40.7\%), 2,989 (37.7\%); LDL cholesterol: 21,487 (33.6\%), 15,665 (41.3\%), 12,605 (42.0\%), 3,060 (38.6\%); and triglycerides: 21,459 (33.6\%), 15,605 (41.1\%), 12,550 (41.8\%), 3,055 (38.6\%)

'Proportion was calculated using the number of patients with available data as the denominator 
Table 2 Distribution of study cohort patients based on key REWIND CV risk criteria

\begin{tabular}{ll}
\hline Distribution of study cohort patients & Values \\
\hline Total T2D study cohort, $\boldsymbol{N}(\%)$ & $\mathbf{6 3 , 9 2 7}(\mathbf{1 0 0 . 0})$ \\
Patients fulfilling key REWIND CV criteria, total, $\boldsymbol{n}(\mathbf{\%})^{\mathrm{a}}$ & $\mathbf{3 7 , 9 6 5 ( 5 9 . 4 )}$ \\
Patients fulfilling key REWIND CV criteria with history of established CVD, $\boldsymbol{n}(\mathbf{\%})$ & $\mathbf{7 9 2 2 ( 1 2 . 4 )}$ \\
Prior myocardial infarction & $2609(4.1)$ \\
Ischemic stroke & $2081(3.3)$ \\
Presence of coronary revascularization & $1970(3.1)$ \\
Occlusion of stenosis of precerebral arteries, carotid artery & $1801(2.8)$ \\
Hospitalization for unstable angina (approximation) & $963(1.5)$ \\
Patients fulfilling key REWIND CV criteria without established CVD, $\boldsymbol{n}(\mathbf{\%})^{\mathrm{b}}$ & $\mathbf{3 0 , 0 4 3}(\mathbf{4 7 . 0})$ \\
$\geq 55$ years with $\geq 1$ subclinical vascular disease & $6205(9.7)$ \\
$\geq 60$ years with $\geq 2$ CV risk factors & $28,554(44.7)$ \\
\hline
\end{tabular}

Data presented as the number $(\mathrm{n})$ in and percentage of the total study cohort $(N)$

$C V$ Cardiovascular, $C V D$ cardiovascular disease; $N$ number of patients

${ }^{\text {a}}$ Proxies were used to apply REWIND CV criteria

${ }^{b}$ Patients could belong to both strata

$7.1 \%$ and mean BMI was $30.4 \mathrm{~kg} / \mathrm{m}^{2}$ ( \pm 5.8$)$. The most frequent comorbidities reported by the physician were hypertension $(63.8 \%$ of patients) and hyperlipidemia (31.4\%). An additional analysis considering diagnosis and prescriptions has shown that $78.2 \%$ of the general T2D population had hypertension and $63.7 \%$ had hyperlipidemia. The mean $( \pm$ SD) age of REWIND participants was $66.2( \pm 6.5)$ years and $53.7 \%$ were male; mean $\mathrm{HbA} 1 \mathrm{c}$ was $7.3 \%$ and mean BMI was $32.3( \pm 5.7) \mathrm{kg} / \mathrm{m}^{2}$.

\section{Patient Characteristics Based on Key REWIND CV Criteria}

Of the total T2D study population, 37,965 $(59.4 \%)$ were aged $\geq 50$ years and fulfilled the REWIND CV criteria (Table 2). More specifically, 7922 (12.4\%) of the total T2D study population were aged $\geq 50$ years and had a history of established CVD (Table 2), which represents $20.9 \%$ of the T2D patients who fulfilled REWIND CV criteria (7922 of the 37,965$)$. The majority of these patients, 7751 (97.8\%), had one or two prior CVDs.

In addition, 30,043 (47\%) of the total T2D study population fulfilled REWIND CV criteria without a history of established CVD (Table 2). Specifically, $6205(9.7 \%)$ were aged $\geq 55$ years with $\geq 1$ subclinical vascular disease and 28,554 $(44.7 \%)$ were aged $\geq 60$ years with $\geq 2 \mathrm{CV}$ risk factors. Patients could belong to both strata. Of the patients aged $\geq 60$ years with $\mathrm{CV}$ risk factors, $26,927(94.3 \%)$ had two or three CV risk factors.

Baseline characteristics of the population who satisfied REWIND CV criteria, those with established CVD and those without are presented alongside those from the REWIND study in Table 1. In the population fulfilling REWIND $\mathrm{CV}$ criteria, age was comparable between patients with or without established CVD, whereas gender and BMI were not, with more males in the population with established CVD and a higher BMI in the population without established CVD. The eGFR was similar regardless of $\mathrm{CV}$ profile although a higher proportion 
a

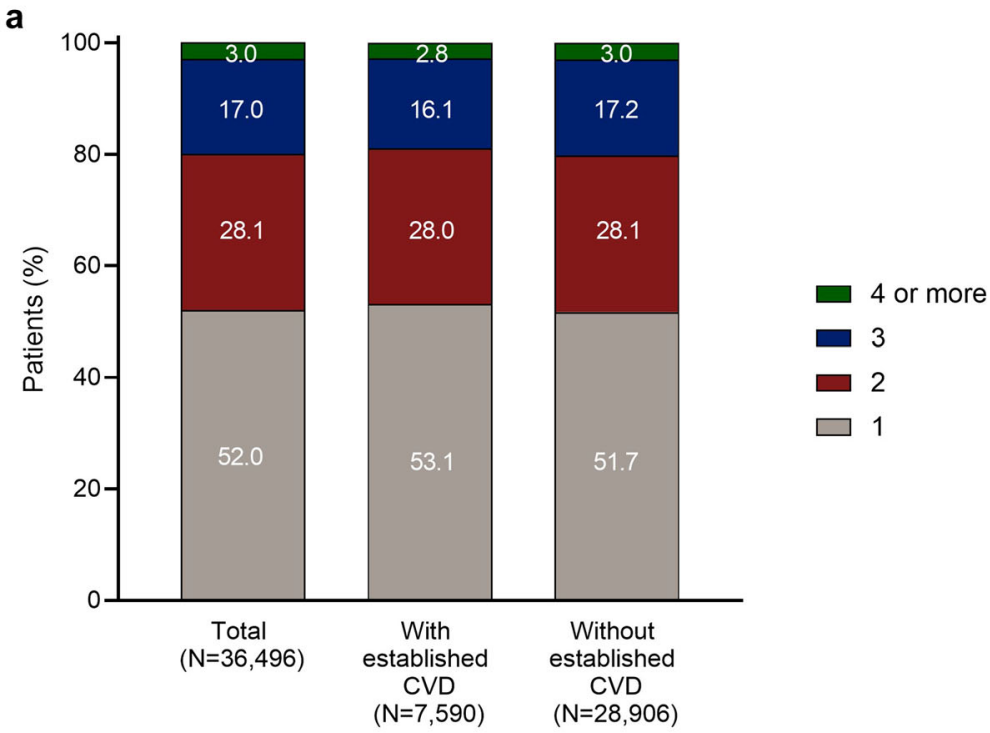

b

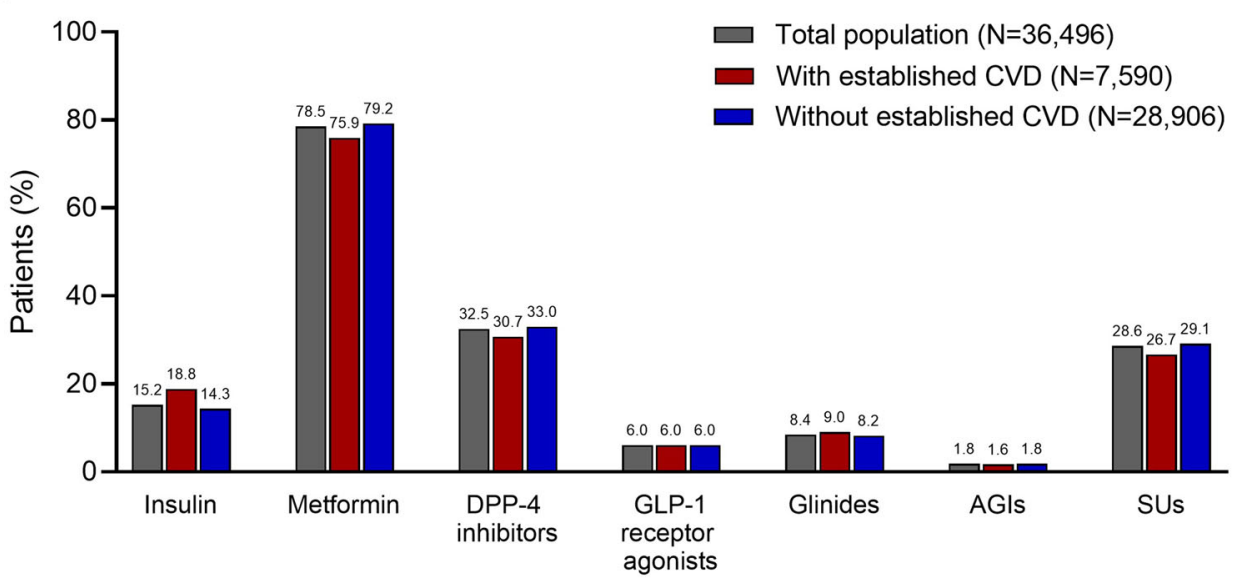

Fig. 2 Antidiabetic treatment patterns of patients who satisfied key REWIND CV criteria $(N=37,965)$ receiving at least one antidiabetic treatment. a The percentage of patients receiving $1,2,3$, or $\geq 4$ antidiabetic treatments in the total population, the population with established CVD and the population without established CVD. $\mathbf{b}$ Types of

of patients with established CVD had an estimated glomerular filtration rate $\leq 60 \mathrm{ml} / \mathrm{min} /$ $1.73 \mathrm{~m}^{2}$. A slightly higher percentage of patients with CVD had diagnosed albuminuria than those without CVD. HbA1c was similar between the two subgroups and patients with established CVD had lower low-density lipoprotein-cholesterol levels. oral or injectable antidiabetic treatments that the total population, and patients with and without established CVD received. AGIs Alpha glucosidase inhibitors, CVD cardiovascular disease, DPP-4 dipeptidylpeptidase-4, $G L P-1$ glucagon like peptide-1, $S U$ s sulfonylureas

\section{Treatment Patterns of Patients With and Without Established CVD Meeting REWIND CV Criteria}

In the population fulfilling key REWIND CV criteria, $7590(95.8 \%)$ and 28,906 (96.2\%) of the patients with and without established CVD, respectively, received at least one GLA. In both groups, over $50 \%$ of patients received only one GLA (Fig. 2a). Metformin was the most 
prescribed GLA, while $18.8 \%$ of the patients with established CVD and $14.3 \%$ of the patients without established CVD received insulin (Fig. 2b). The most common treatment regimen was metformin alone, with $34.5 \%$ and $36.1 \%$ of the populations with and without established CVD receiving metformin alone, respectively (ESM Fig. 1). GLP-1 RAs were prescribed to 6\% of patients, regardless of their CVD status (Fig. 2b).

Among the patients who fulfilled key REWIND criteria, $91.1 \%$ and $87.3 \%$ of patients with and without established CVD, respectively, received blood pressure medications. Statin use was also prevalent among the populations, with $70.5 \%$ and $62.6 \%$ of patients with and without established CVD, respectively, prescribed a statin. Fibrate use was low, with $2.4 \%$ and $6.8 \%$ of patients with and without established CVD, respectively, receiving fibrate medications (ESM Fig. 2).

\section{DISCUSSION}

This study described patients with T2D followed by GPs in routine clinical practice in France and put them in the perspective of patients included in the REWIND study cohort, applying key REWIND inclusion criteria to examine patients' $\mathrm{CV}$ risk profiles. While many baseline characteristics were comparable, others, including duration of diabetes, the presence of hypertension and albuminuria, differed between the two cohorts. After applying REWIND CV inclusion criteria, some baseline characteristics of the study cohort notably changed, such as mean duration of diabetes, the percentage of patients with hypertension and eGFR $<60 \mathrm{ml} / \mathrm{min} /$ $1.73 \mathrm{~m}^{2}$. The percentage of patients with albuminuria was low compared to the REWIND cohort $(2.4 \%$ of the total T2D population fulfilling key REWIND criteria versus $35 \%$ of the REWIND study cohort), but the prevalence presented here may be biased as albuminuria: (1) was recorded as a categorical variable within the LPD database, which did not allow differentiating the effective absence of albuminuria and missing values; (2) may have been underreported by physicians in the database; and (3) is not often assessed in routine practice. Indeed, the ENTRED 2007 report found that only 28\% of patients had a dosage of albuminuria or proteinuria within the previous year, and albuminuria status was not reported for $39 \%$ of patients, which does not allow us to assess the prevalence of albuminuria in French patients with T2D [11, 12]. In addition, in an effort to encourage the monitoring of renal function in patients with T2D and improve quality of care, the National Health Insurance Fund (Caisse Nationale d'Assurance Maladie) has recently included an annual albuminuria threshold as a quality indicator within the French primary care pay-for-performance program [13].

Previous studies comparing the eligibility of patients with T2D in routine clinical care with major phase 3 randomized controlled trials (including GLP-1 RA and sodium-glucose cotransporter-2 (SGLT2) inhibitor CVOTs) have shown limited eligibility of general populations based on trial inclusion-exclusion criteria and differences between baseline characteristics [14-18]. The proportion of patients in general populations meeting CVOT eligibility ranged from $16.6 \%$ of an English general T2D population for the LEADER CVOT to 59\% of a pooled German, Dutch, Swedish and Norwegian general T2D population for the DECLARE-TIMI 58 CVOT $[16,18]$. Similarly, a Scottish study that examined the proportion of patients with T2D in clinical care who met the eligibility criteria of seven major T2D randomized controlled trials which have been used to form diabetes treatment guidelines for glycemic control (ACCORD, ADVANCE, PROactive, RECORD, VADT, UKPDS 33 and UKPDS 34) found that a maximum of $51 \%$ of newly diagnosed patients were eligible for these trials, with age and renal impairment being two of the most common reasons for exclusion [15]. Additionally, heterogeneity between cohorts from different countries has been published [14, 18], necessitating studies to understand how different CVOT criteria apply to individual countries. While the baseline characteristics of the REWIND CVOT were suggested to be the most generalizable to patients with $\mathrm{T} 2 \mathrm{D}$ in routine clinical practice in the USA from four major GLP-1 RA CVOTs [8], it was of interest to analyze the eligibility of the French patients to the 
REWIND study as participants were neither recruited to the REWIND trial nor studied in France [9]. The current study showed that a large proportion of the French study population were eligible for REWIND, with $59.4 \%$ of the general study population fulfilling REWIND CV criteria, including $12.4 \%$ with established CVD. The lower percentage of patients with established CVD than in the REWIND study (31.4\%) [10] was expected as patients enrolled in CVOTs are usually highly selected for very high CV risk.

The prevalence of CVD in patients with T2D in routine clinical care varies depending on the study design and the region [19]. Among European studies $[18,20-22]$, the prevalence of CVD has been reported to range from 6.9 [22] to $44 \%$ [18], although different patient inclusion criteria and definitions of CVD may contribute to these differences. Among the French population in $2007,26 \%$ of patients with T2D had a high risk of CVD and $16.7 \%$ of patients reported angina or myocardial infarction while 13.9\% reported coronary revascularization [11]. The CAPTURE study, conducted over 10 months between 2018 and 2019, analyzed 659 French patients with T2D within a total cohort of 9823 patients across 13 countries; the results indicated a $34.2 \%$ prevalence of CVD among French patients, similar to the overall cohort [19]. This result is much higher than the current analysis in which $12.4 \%$ of patients had a history of CVD; however, there is a large difference in sample sizes between the studies, and the CAPTURE study population was recruited almost equally from primary care and specialists settings [11]. In another study conducted in 2011 on the National Health Insurance CrossSchemes Information System (SNIIRAM) data, $26 \%$ of patients with diabetes in France received care for a cardio-neurovascular disease (coronary disease, ischemic stroke, heart failure, lower extremity arterial disease, cardiac arrythmia, valvular disease, acute pulmonary embolism or another CV condition) [23]. The same study showed that the average age of the French population with diabetes was 65 years and that $48 \%$ were women. While this slightly contrasts the current analysis in which the average age of patients was 67 years and $42 \%$ were women, the differences may be explained by the gap in study dates and the fact that the SNIIRAM study did not differentiate between patients with type 1 diabetes and T2D. Finally, the 2007 ENTRED study reported that $41 \%$ of patients were obese [11], compared to $47.6 \%$ in the current study population and $61.2 \%$ in REWIND study. Nonetheless, the current analysis is largely similar to reported results for French patients in 2007 and 2011 [19].

The majority of patients in this study received metformin $(75.9 \%$ and $79.2 \%$ of patients with and without established CVD, respectively), which was comparable to the REWIND study in which $81 \%$ of patients received metformin at baseline, and also in accordance with French guidelines for the management of hyperglycemia in T2D [24]. The percentages of patients receiving insulin were somewhat lower in the current study than in the REWIND study, with $18.8 \%$ and $14.3 \%$ of the populations with or without established CVD, respectively, and approximately $24 \%$ of REWIND participants receiving insulin at baseline. Dipeptidylpeptidase-4 (DPP-4) inhibitors were used by $>30 \%$ of patients fulfilling key REWIND criteria. In REWIND, patients were instructed to remain on their antihyperglycemic therapy, with the exception of patients taking a DPP-4 inhibitors or GLP-1 RAs at screening, who discontinued these therapies at the start of the run-in period [10]. Glinide usage was also higher in the current study, while sulfonylurea usage was higher in the REWIND study, and $\alpha$-glucosidase inhibitor usage was similar between both. The differences in treatment patterns between the current and the REWIND studies could be due to geographical differences in patient characteristics, treatment guidelines and sources of participants recruitment (primary vs. tertiary care).

The pattern of glucose-lowering medication usage was comparable between subgroups of patients with and without established CVD, as defined by key REWIND criteria, which was consistent with the treatment pattern observed in the CAPTURE study. Despite the prevalence of CVD and CV risk factors among the general T2D study population, $6 \%$ of patients fulfilling REWIND CV criteria were prescribed GLP-1 RAs, regardless of their $\mathrm{CV}$ profile and frequently in 
addition to metformin alone or metformin and sulfonylureas. This percentage is lower than that described by the CAPTURE study which reported that $8.6 \%$ of participants worldwide and $24.7 \%$ of French participants were prescribed a GLP-1 RA with a similar usage across patients with and without CVD [19, 25]. This may be explained by the fact that of the 659 French participants in the CAPTURE study, 361 (54.8\%) were followed by a GP and 298 by a specialist (45.2\%) [19]; and GLP-1 RAs are more frequently prescribed by specialists than GPs (41.9 vs. 8.6\%) [25]. Given that the current study only analyzed patients under GP care, this may account for the difference with CAPTURE data in GLP-1 RA prescriptions. However the absence of a difference in prescription according to patient's CV profile was observed in both studies [26-29].

This study has several strengths. It used a large and robust cohort that is representative of the general French patient population. The data source collected information from a panel of 1200 GPs in a non-interventional way using pseudonymized, patient-level information that reflects routine clinical practice. The panel of contributing GPs was maintained as a representative sample of primary care physicians according to three criteria known to influence prescriptions: age, gender and geographical distribution. Whenever a physician left the panel, they were replaced by another one with a similar profile. Indeed, public health data have previously highlighted that $82 \%$ of patients with T2D are routinely followed-up by a GP only and that only $14 \%$ had an appointment with a private specialist physician throughout a 2-year period [12].

The study also has several limitations. There were a high number of missing values (as indicated in Table 1), and it was impossible to query the physicians for any missing information as no individual patient identifiers were available. The analyzed data did not capture data from patients managed exclusively by specialists. To apply REWIND CV criteria, some information was approximated with proxies using data available in the IQVIA LPD database for France. Certain study variables were underreported in the EMRs, including weight, laboratory values and smoking status (see Table 1; smoker status unknown, 98.7\%). In some situations, proxies used a combination of several indicators and may have caused an overestimation. Due to Commission Nationale de l'Informatique et des Libertés (CNIL) legal requirements, only the previous 10 years could be studied, which may have affected some results, especially T2D duration. Within secondary outcomes, CV risk groups were not exclusive, meaning patients could belong to several groups, which leads to an overestimation of the proportion of patients in each stratum. In patients aged $\geq 55$ years with subclinical vascular disease, documented myocardial ischemia by stress tests or imaging and $\geq 50 \%$ coronary carotid or lower extremity artery stenosis were included in the REWIND criteria; however, this information was not available in the current study, so some patients may have been incorrectly excluded. The absence of French patients in REWIND limits the transposability of the results to French patients with T2D but highlights the relevance of our study.

\section{CONCLUSIONS}

In conclusion, this observational study of 63,927 French patients with T2D showed that the mean age, HbA1c and BMI observed were comparable to the REWIND population. Almost $60 \%$ of the general study population our this observational study fulfilled REWIND criteria. A lower percentage of patients aged $\geq 50$ years had established CVD in this cohort than that in the REWIND study. Despite their benefits for lowering CVD risk, also highlighted in international T2D management guidelines, GLP-1 RAs were infrequently prescribed, regardless of the participants' established CVD status. Future studies should investigate if recent re-assessments of GLP-1 RAs impact the prescription of these drugs in T2D patients with and without a history of CVD. 


\section{ACKNOWLEDGEMENTS}

Funding. This work and journal's Rapid Service Fee was funded by Eli Lilly and Company.

Authorship. All named authors meet the International Committee of Medical Journal Editors (ICMJE) criteria for authorship for this article, take responsibility for the integrity of the work as a whole, and have given their approval for this version to be published.

Author Contributions. Conceptualization: AD. Design of work: AD, FC, KI, IJ. Data acquisition: FC. Formal analysis and investigation: $\mathrm{AD}, \mathrm{FC}, \mathrm{KI}, \mathrm{IJ}, \mathrm{LL}, \mathrm{PD}$. Writing review: AD, FC, KI, IJ, LL, PD.

Medical Writing, Editorial, and Other Assistance. The authors would like to thank Ana Hickey, Eli Lilly and Company, for medical writing and editorial services, and IQVIA for assistance with data analysis.

Prior Presentation. Partial data from this study were presented at Société Francophone du Diabète, 23-26 March 2021.

Disclosures. Anne Dib, Florence Chartier, Katharina Ihle, Isabelle Jammoneau and Lisa Ludwig are employees of Eli Lilly and Company. Patrice Darmon reports honoraria, consultancy, advisory role, lectures or travel grants from AstraZeneca, Boehringer Ingelheim, Eli Lilly and Company, Merck Sharp and Dohme, Novartis, Novo Nordisk, Sanofi, Mundipharma, and Abbott.

Compliance with Ethics Guidelines. This study is based on de-identified data collected from a healthcare database. According to the French law no submission to CESREES (Ethics and Scientific Committee for Research, Studies and Evaluations in the Field of Health) was required. This study complied with the reference method MR004 of CNIL (French data privacy agency). As required, the study was recorded for information on the French Health Data Hub website.

Data Availability. The original de-identified data used in this analysis were obtained from and are the property of IQVIA. IQVIA has restrictions prohibiting the authors from making the dataset publicly available. Interested researchers may contact IQVIA France to apply to gain access to the study's data directly (see https://www.iqvia.com/fr-fr/contact) or from the corresponding author upon reasonable request.

Open Access. This article is licensed under a Creative Commons Attribution-NonCommercial 4.0 International License, which permits any non-commercial use, sharing, adaptation, distribution and reproduction in any medium or format, as long as you give appropriate credit to the original author(s) and the source, provide a link to the Creative Commons licence, and indicate if changes were made. The images or other third party material in this article are included in the article's Creative Commons licence, unless indicated otherwise in a credit line to the material. If material is not included in the article's Creative Commons licence and your intended use is not permitted by statutory regulation or exceeds the permitted use, you will need to obtain permission directly from the copyright holder. To view a copy of this licence, visit http://creativecommons.org/licenses/by$\mathrm{nc} / 4.0 /$.

\section{REFERENCES}

1. Shah AD, Langenberg C, Rapsomaniki E, Denaxas $\mathrm{S}$, Pujades-Rodriguez M, Gale CP, et al. Type 2 diabetes and incidence of cardiovascular diseases: a cohort study in 1-9 million people. Lancet Diabetes Endocrinol. 2015;3(2):105-13.

2. Kristensen SL, Rørth R, Jhund PS, Docherty KF, Sattar N, Preiss D, et al. Cardiovascular, mortality, and kidney outcomes with GLP-1 receptor agonists in patients with type 2 diabetes: a systematic review and meta-analysis of cardiovascular outcome trials. Lancet Diabetes Endocrinol. 2019;7(10):776-85. 
3. Hernandez AF, Green JB, Janmohamed S, D'Agostino RB, Granger CB, Jones NP, et al. Albiglutide and cardiovascular outcomes in patients with type 2 diabetes and cardiovascular disease (Harmony Outcomes): a double-blind, randomised placebocontrolled trial. Lancet. 2018;392(10157):1519-29.

4. Holman RR, Bethel MA, Mentz RJ, Thompson VP, Lokhnygina Y, Buse JB, et al. Effects of once-weekly exenatide on cardiovascular outcomes in type 2 diabetes. N Engl J Med. 2017;377(13):1228-39.

5. Marso SP, Bain SC, Consoli A, Eliaschewitz FG, Jódar E, Leiter LA, et al. Semaglutide and cardiovascular outcomes in patients with type 2 diabetes. N Engl J Med. 2016;375(19):1834-44.

6. Marso SP, Daniels GH, Brown-Frandsen K, Kristensen P, Mann JFE, Nauck MA, et al. Liraglutide and cardiovascular outcomes in type 2 diabetes. N Engl J Med. 2016;375(4):311-22.

7. Pfeffer MA, Claggett B, Diaz R, Dickstein K, Gerstein HC, Køber LV, et al. Lixisenatide in patients with type 2 diabetes and acute coronary syndrome. N Engl J Med. 2015;373(23):2247-57.

8. Boye KS, Riddle MC, Garcia-perez RML, Lage MJ, Riesmeyer JS, Lakshmanan MC. Generalizability of glucagon-like peptide-1 receptor agonist cardiovascular outcome trials to the overall type 2 diabetes population in the United States. Diabetes Obes Metab. 2019;21(6):1299-304.

9. Gerstein HC, Colhoun HM, Dagenais GR, Diaz R, Lakshmanan M, Pais $P$, et al. Dulaglutide and cardiovascular outcomes in type 2 diabetes (REWIND): a double-blind, randomised placebo-controlled trial. Lancet. 2019;394(10193):121-30.

10. Gerstein HC, Colhoun HM, Dagenais GR, Diaz R, Lakshmanan M, Pais P, et al. Design and baseline characteristics of participants in the Researching cardiovascular Events with a Weekly INcretin in Diabetes ( REWIND) trial on the cardiovascular effects of dulaglutide on behalf of the REWIND Trial Investigators. Diabetes Obes Metab. 2018;20(1):42-9.

11. Fagot-Campagna A, Fosse S, Roudier C, Romon I, Penfornis A, Lecomte $\mathrm{P}$, et al. Characteristics, vascular risk and complications in people with diabetes, in metropolitan France: major improvements between ENTRED 2001 and ENTRED 2007 studies. Bull Epidemiol Hebd. 2009;42/43:450-5.

12. Druet C, Bourdel-Marchasson I, Weill A, Eschwege E, Penfornis A, Fosse $S$, et al. Le diabète de type 2 en France: épidémiologie, évolution de la qualité de la prise en charge, poids social et économique. ENTRED 2007. Presse Med. 2013;42(5):830-8.
13. Caisse Nationale d'Assurance Maladie (CNAM) CNAM guide methodologique Rémunération sur Objectifs de Santé Publique (ROSP); 2020. https:// www.ameli.fr/paris/medecin/exercice-liberal/ remuneration/remuneration-objectifs/medecintraitant-adulte.

14. Castellana M, Procino F, Sardone R, Trimboli P, Giannelli G. Generalizability of sodium-glucose cotransporter-2 inhibitors cardiovascular outcome trials to the type 2 diabetes population: a systematic review and meta-analysis. Cardiovasc Diabetol. 2020;19(1):87.

15. Saunders C, Byrne CD, Guthrie B, Lindsay RS, McKnight JA, Philip S, et al. External validity of randomized controlled trials of glycaemic control and vascular disease: how representative are participants? Diabet Med. 2013;30(3):300-8.

16. Hinton $\mathrm{W}$, Feher $\mathrm{M}$, Munro $\mathrm{N}$, Walker $\mathrm{M}$, de Lusignan S. Real-world prevalence of the inclusion criteria for the LEADER trial: data from a national general practice network. Diabetes Obes Metab. 2019;21(7):1661-7.

17. Arnold SV, Inzucchi SE, Tang F, Mcguire DK, Mehta SN, Maddox TM, et al. Real-world use and modeled impact of glucose-lowering therapies evaluated in recent cardiovascular outcomes trials: an NCDR Research to Practice project. Eur J Prev Cardiol. 2017;24(15):1637-45.

18. Birkeland KI, Bodegard J, Norhammar A, Kuiper JG, Georgiado E, Beekman-Hendriks WL, et al. How representative of a general type 2 diabetes population are patients included in cardiovascular outcome trials with SGLT2 inhibitors? A large European observational study. Diabetes Obes Metab. 2019;21(4):968-74.

19. Mosenzon O, Alguwaihes A, Leon JLA, Bayram F, Darmon P, Davis TME, et al. CAPTURE: a multinational, cross-sectional study of cardiovascular disease prevalence in adults with type 2 diabetes across 13 countries. Cardiovasc Diabetol. 2021;20(1):154.

20. Eliasson B, Ekelund J, Amberntsson R. Cardiovascular disease in patients with type 2 diabetes and in patients starting empagliflozin treatment: nationwide survey. Diabetes Ther. 2019;10(4):1523-30.

21. Lautsch D, Wang T, Yang L, Rajpathak SN. Prevalence of established cardiovascular disease in patients with type 2 diabetes Mellitus in the UK. Diabetes Ther. 2019;10(6):2131-7.

22. Artime E, Romera I, Díaz-Cerezo S, Delgado E. Epidemiology and economic burden of cardiovascular disease in patients with type 2 diabetes Mellitus in Spain: a systematic review. Diabetes Ther. 2021;12(6):1631-59. 
23. Cosker $K$, Denis $P$, Tala $S$, Rigault $A$, Rivière $S$, Pestel $\mathrm{L}$, et al. Pathologies associées, états de santé et traitements des personnes diabétiques en France: Les données du Sniiram. Bull Epidémiol Hebd. 2011;2014:507-14.

24. Haute Autorité de Santé (HAS). Stratégie médicamenteuse du contrôle glycémique du diabète de type 2. Saint-Denis La Plaine: HAS. 2013.

25. Darmon P, Meyer L, Marcilla A, Jubin L. Utilisation des médicaments cardioprotecteurs chez les sujets atteints de diabète de type 2 en France en 2019: résultats de 1 ' étude CAPTURE. Presentation at the Société Francoph du Diabète Congrès Annu, 23-26 March 2021, Strasbourg.

26. Arnett DK, Blumenthal RS, Albert MA, Buroker AB, Goldberger ZD, Hahn EJ, et al. 2019 ACC/AHA guideline on the primary prevention of cardiovascular disease: a report of the American College of Cardiology/American Heart Association Task Force on clinical practice guidelines. Circulation. 2019;140(11):e596-646.

27. American Diabetes Association. 6. Glycemic targets: standards of medical care in diabetes-2021. Diabetes Care. 2021;44(Suppl. 1):S73-84.

28. Cosentino F, Grant PJ, Aboyans V, Bailey CJ, Ceriello A, Delgado V, et al. 2019 ESC Guidelines on diabetes, pre-diabetes, and cardiovascular diseases developed in collaboration with the EASD: the Task Force for diabetes, pre-diabetes, and diseases of the European Society of Cardiology (ESC) and the European Associ. Eur Heart J. 2020;41(2):255-323.

29. Garber AJ, Handelsman Y, Grumberger G, Einhorn D, Abrahamson MJ, Barzilay JI, et al. Consensus statement by the American Association of Clinical Endocrinologists and American College of Endocrinology on the comprehensive type 2 diabetes management algorithm-2020 executive summary. Endocr Pract. 2020;26(1):107-39. 